\title{
The Upgrade for the Data Acquisition System of the KOTO Detector
}

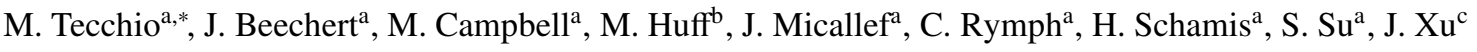 \\ ${ }^{a}$ University of Michigan, Ann Arbor, MI, U.S.A. \\ ${ }^{b}$ Kenyon College, Gambier, OH, U.S.A. \\ ${ }^{c}$ Apple Inc., Cupertino, CA, U.S.A.
}

\begin{abstract}
A major upgrade to the KOTO detector data acquisition system based on the ATCA standard is being considered. The ATCA standard provides a natural solution to the current KOTO constraints, including communication between boards and higher input and output bandwidth.
\end{abstract}

Keywords: Trigger, DAQ, KOTO, ATCA

\section{Introduction}

The goal of KOTO experiment [1] at J-Parc is to discover and ${ }_{34}{ }_{33}$ measure the rate of the rare decay $K_{L} \rightarrow \pi^{0} v \bar{v}$, whose branching ${ }_{35}$ ratio is predicted to be $(2.4 \pm 0.4) \times 10^{-11}$ [2] in the Standard ${ }_{36}$ Model. The KOTO data acquisition system (DAQ) [3] is built ${ }_{37}$ around a 14-bit 125MHz ADC frontend board and a three-tiered ${ }_{38}$ trigger system. The analog signals from $3000 \mathrm{CsI}_{\text {calorimeter }}{ }_{39}$ channels and 1000 veto detector channels are shaped, digitized and stored inside a $4 \mu$ s deep pipeline. The first two levels of trigger are implemented in custom-made $9 \mathrm{U}$ VME boards re- 40 ceiving data from the ADCs via 2 Gbps fiber links. Events passing the Lv1 and Lv2 decision are sent to a computer farm via 41 a Gibagit Ethernet connection for event reconstruction and Lv3 42 trigger. This system was used during KOTO first physics run 43 in May 2013 and ran at sustained Lv1 and Lv2 trigger rates of 44 $14 \mathrm{kHz}$ and $3.8 \mathrm{kHz}$, respectively, as per design specifications. 45

In this paper we will first review the performance of the 46 KOTO DAQ in the current configuration and then present a se- 47 ries of upgrades aimed at coping with the increase in proton 48 beam intensity projected for 2016 and 2017 . These upgrades 49 will allow to process data rates up to $100 \mathrm{kHz}$ at Lv1 with min- 50 imal dead time while increasing the capacity of the system.

\section{DAQ Performance}

The J-Parc Main Ring synchrotron accelerates protons to ${ }^{55}$ $30 \mathrm{GeV}$ and provides a high intensity beam to the KOTO detec- ${ }^{56}$ tor using a slow extraction technique. During the first 100-hour ${ }^{57}$ physics run in May 2013, the single beam extraction, or spill, ${ }^{58}$ had a duration of 2 second and a repetition rate of 6 second, with ${ }^{59}$ sustained peak beam intensity of $24 \mathrm{~kW}$. In these running con- ${ }^{60}$ ditions, the KOTO DAQ routinely took data at rates of $\sim 28000^{61}$ events per spill at Lv1 and $\sim 7600$ events at Lv2. Each event ${ }^{62}$

*Corresponding author

Email address: tecchio@umich.edu (M. Tecchio) had a size of $250 \mathrm{~kb}$ before compression. The DAQ livetime, defined as the ratio of events considered for a Lv2 trigger decision vs the total amount of event accepted by the Lv1 trigger, was around $80 \%$, limited by the available buffering at the input and output of Lv2 Trigger board. At the end of the run, KOTO collected enough data to reach a single event sensitivity of $\left(1.29 \times 10^{-8}\right)$ [4], comparable to the sensitivity of the previous best $K_{L} \rightarrow \pi^{0} v \bar{v}$ direct search [5].

\section{DAQ Upgrade}

We performed simulation studies of the DAQ livetime vs the Lv1 trigger rate by optimizing different parameters of the Lv2 and Lv3 trigger design, such as buffering resources, latency, bandwidth and rejection power of different physics-based algorithms. The delivered beam power is expected to reach the $50 \mathrm{~kW}$ to $100 \mathrm{~kW}$ range over next 2 years, with projected Lv1 rates well above $50 \mathrm{kHz}$. We looked into ways to upgrade the present DAQ so that it can sustain such rates while keeping the livetime at the $90 \%$ level or higher

We decided to go for an incremental approach and upgrade different parts of the KOTO DAQ in between physics runs, typically consisting of three weeks of continuous data taking 2-3 times every year. First we upgraded the software based Lv3 trigger from a farm of 29 PC nodes connected via a Gigabit Ethernet switch to a farm of 47 PC nodes running a Real Time Linux operating system and connected via a $10 \mathrm{Gbs}$ Infiniband network. While a subset of the farm is dedicated to read the data from individual Lv2 trigger boards via a $1 \mathrm{Gbs}$ port using UDP protocol, the rest of the farm uses MPI IB verbs for event building and exploits the direct memory access capability and the ability to transfer messages between applications without relying on the operating system that Infiniband offers. This upgrades was successfully commissioned in April 2015.

The next step in the DAQ upgrade focuses on implementing a lossless compression algorithm, able to reduce the data to one third of the original size, directly in the ADC firmware. This 


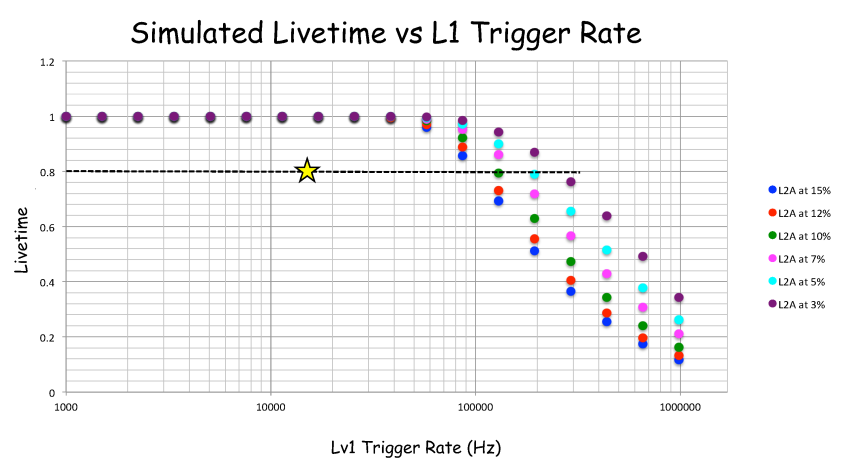

Figure 1: DAQ livetime vs Lv1 trigger rate for Lv2 trigger rejection rates be-102 tween $15 \%$ and $3 \%$, assuming online data with a compression factor of about $30 \%$.. The yellow star shows the present DAQ running point of $80 \%$ livetime at $_{103}$ $14 \mathrm{kHz}$ Lv1 rate achieved with uncompressed data and a typical Lv2 rejection factor of $\sim 30 \%$. requires a redesign of the Lv2 trigger firmware to keep the syn- ${ }^{108}$ chronization of the system during the Lv2 decision, which is based on the energy weigthed average position of the photon ${ }_{109}$ electromagnetic showers in the calorimeter. At the end of this step, the KOTO DAQ will be able to move more efficiently a ${ }^{110}$ larger volume of event, thus improving the livetime.

The online compression will be complemented by upgrades $s_{113}$ to the hardware controlling the generation of the system clock $_{114}$ and the final Lv1 and Lv2 trigger decisions, as well the dis- ${ }_{115}$ tribution of these control signals to the whole DAQ. This will ${ }_{116}$ allow to expand the number of frontend channels as more veto detectors are added to the system. It will also allow to separate events into parallel data streams as they are analyzed by the Lv $3^{117}$ trigger and written to storage.

The final and more ambitious step of the KOTO DAQ up- ${ }_{119}$ grade revolves around a complete redesign of the Lv2 trigger ${ }^{120}$ hardware. We are considering to collaborate with the SLAC ${ }^{121}$ RCE Platform Technology (RPT) project [6] which has devel- ${ }_{123}^{122}$ nology. This system will overcome the present KOTO DAQ ${ }^{125}$ constraints of limited communication between boards and in- ${ }^{126}$ sufficient input and output bandwidth and result in substantially ${ }^{127}$ higher Lv2 trigger rejection rate. In particular, it will allow to use photon counting and detailed timing analysis of calorimeter vs veto signals for the Lv2 trigger decision.

The RPT project has developed an ATCA blade, called Cluster On Board (COB), with bays for up to 9 Reconfigurable Cluster Elements (RCE) for data processing and intercommunication. Each RCE is a bundled set of hardware, firmware and software, built around a Xilinx Zync chip; it supports $12 \times 12.5$ Gbs SERDES, dual core $900 \mathrm{MHz}$ processor plus connections to 64 GB MicroSD Flash, 1 GB DDR3 RAM and native 10Gb Ethernet interface. A Rear Transition Module (RTM) will provide the interface between the RCE and the KOTO data via high speed serial links. oped a generic data acquisition system based on ATCA tech-124

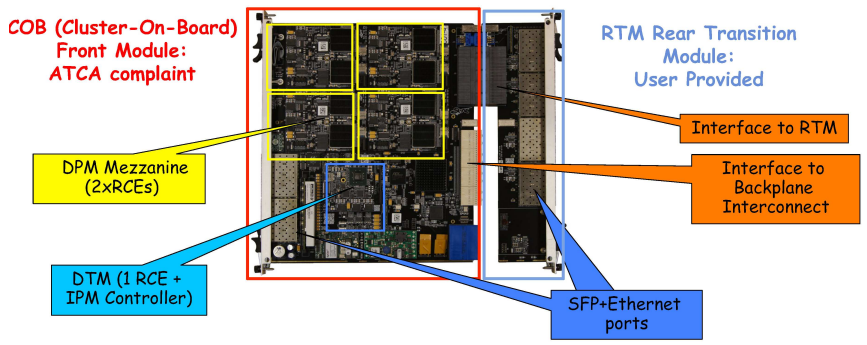

Figure 2: Main features of the COB and RTM boards developed by the SLAC RCE Platform Technology project.

\section{Conclusion}

The main components of the proposed upgrade to the KOTO experiment DAQ are reviewed. A board developed by the RPT SLAC project is being considered as a replacement for the Lv2 trigger electronics. This should allow to keep cope with the projected Level 1 trigger rates expected for an increase in beam intensity up to $100 \mathrm{~kW}$.

\section{Acknowledgments}

The authors would like to express their gratitude to all members of the J-PARC accelerator and Hadron Beam groups for providing stable beam operations; to the members of the KOTO Collaboration for their effort in designing, installing and operating the detector; and for the members of the KOTO DAQ group for providing the results shown in this article. This research was supported US DOE grant DE-SC0002644.

\section{References}

\section{References}

[1] T. Yamanaka for the KOTO collaboration, Prog. Theor. Exp. Phys., 2012, 02B006 (2012).

[2] J. Brod et al., Phys. Rev. D, 83, 034030 (2011).

[3] Y. Sugiyama, et al., IEEE Trans. Nucl. Sci. 62 (2015) 1115-1121.

[4] K. Shiomi, $k_{L} \rightarrow \pi v \bar{v}$ at KOTO, arXiv:1411.4250 [hep-ex].

[5] J. Ahn et al., Phys. Rev. D, 81, 072004 (2010).

[6] R. Herbst et al., 'Design of the SLAC RCE Platform: A general purpose ATCA based data acquisition system", NSS/MIC 2014 / RTSD 2014 Proceedings, SLAC-PUB-16182 (2015). 